\title{
Atención Primaria en Salud en Paraguay: panorámica y perspectiva
}

\author{
Primary Healthcare in Paraguay: overview and prospects
}

\author{
Roberto Dullak ${ }^{1}$ \\ María Isabel Rodriguez-Riveros ${ }^{2}$ \\ Ivani Bursztyn ${ }^{3}$ \\ Maria Stella Cabral-Bejarano ${ }^{4}$ \\ Monica Ruoti ${ }^{2}$ \\ Maria Elsa Paredes ${ }^{4}$ \\ Carmen Wildberger ${ }^{5}$ \\ Faustina Molinas ${ }^{6}$
}

${ }^{1}$ Facultad de Ciencias

Médicas, Universidad Nacional de Asunción. Dr. José P. Montero 658 esquina Dr. Mario Mazzei, Asunción, Paraguay. rdullak@yahoo.com

${ }^{2}$ Instituto de Investigaciones en Ciencias de la Salud, Universidad

Nacional de Asunción.

${ }^{3}$ Departamento de Medicina

Preventiva, Instituto de

Estudos em Saúde Coletiva,

Faculdade de Medicina,

Universidade Federal do

Rio de Janeiro.

${ }^{4}$ Ministerio de Salud

Publica y Bienestar Social, Asunción, Paraguay.

${ }^{5}$ Centro Educativo

Superior en Salud, Ypacaraí,

Paraguay.

${ }^{6}$ Instituto Dr. Andrés

Barbero, Universidad

Nacional de Asunción.
Abstract This paper presents an overview of Primary Health Care (PHC) in Paraguay, as part of a multicentric study, seeking to identify the possibilities of $\mathrm{PHC}$ as a factor for reorganizing the health system. The methodology adopts the comprehensive PHC concept, and takes into consideration the system's segmentation, formed by the public sector of the Ministry of Health and Welfare, Social Security, a not-for-profit private sector, a private for-profit sector and a mixed sector. The study analyzes 5 dimensions: stewardship, financing, resources, integration/continuity, intersectorality/participation, through reviewing data from the literature and official documents, and key informant interviews (experts, decision-makers, professionals and civil society). Advances in health policy legislation since the 1990, with gratuity for all age groups, have been observed. Public spending on health is among the lowest in Latin America. PHC is provided through different vertical programs, with poor coordination and articulation, though a recent political shift prioritizes the progressive implementation of Family Health teams. In conclusion, PHC can contribute to improve health, equity and participation.

Key words Primary Healthcare, Health systems, Paraguay
Resumen En este artículo se presentan una visión panorámica de la Atención Primaria en Salud (APS) en Paraguay, parte de un estudio multicéntrico para identificar las posibilidades de la APS como reordenador del sistema. Adopta el concepto de APS abarcadora y lleva en consideración la segmentación del sistema, conformado por: el sector público del Ministerio de Salud Pública y Bienestar Social, el Seguro Social (Instituto de Previsión Social), el sector privado sin fines de lucro, privado con fines de lucro y mixto. Analiza cinco dimensiones: rectoría, financiamiento, recursos, integración/continuidad, intersectorialidad/participación, por medio de la revisión de literatura, documentos oficiales, y entrevistas con actores claves (expertos, gestores, profesionales y sociedad civil). Visualiza avances en el marco jurídico sanitario desde los años 1990, orientado a la gratuidad en todos los grupos de edad, concretizada en 2008. El gasto público en salud está entre los más bajos de América Latina. La APS se desarrolla en diferentes programas verticales, con escasa coordinación y articulación, pero un cambio reciente en la política prioriza la instalación progresiva de equipos de Salud Familiar. En conclusión, la APS puede contribuir para aumentar la salud, equidad y participación.

Palabras clave Atención primaria a la Salud, Sistemas de Salud, Paraguay 


\section{Introducción}

En este artículo se presentan datos de la investigación realizada en Paraguay como parte del "Estudio multicéntrico en atención primaria en salud: modelos asistenciales, integración al sistema de salud e intersectorialidad en contextos urbanos en Argentina, Brasil, Paraguay y Uruguay" coordinado por la Red de Investigación en Sistemas y Servicios de Salud en el Cono Sur y financiado por el International Development Research Center (IDRC). El objetivo del estudio fue identificar límites y posibilidades para reorientar la atención primaria en salud como estrategia para alcanzar sistemas de salud integrados y universales, considerando el grado de segmentación del sistema de salud y la fragmentación en la provisión de los servicios ${ }^{1}$.

Paraguay tiene dos regiones naturales, divididas por el río Paraguay: la región Oriental, con el $39 \%$ del territorio y algo más del $97 \%$ de la población; y la región Occidental o Chaco, con el 61\% del territorio y un poco más del $2 \%$ de la población. En el Tabla 1 se presentan algunos indicadores seleccionados para describir lo contexto del país. Con un total de 6.054.976 de habitantes; los jóvenes constituyen la población mayoritaria con un $37,1 \%$ de menores de 15 años, situación más acentuada en las áreas rurales $(41,3 \%)$ que en las urbanas $(33,9 \%)$, debido a la tardía entrada al proceso de transición demográfica. La población económicamente activa (15 a 64 años), oscila alrededor del 60\%. Los habitantes mayores de 60 años, corresponden al 7,1\% de la población total (Tabla 1). La población indígena representa al $1,7 \%$ de la población total del país ${ }^{2}$ y se distribuye homogéneamente, con poco más de la mitad $(52,5 \%)$ en la región Oriental y el resto $(47,5 \%)$ en la región Occidental. La estructura por edad muestra una población eminentemente joven y con escasa población adulta. En promedio, esta población cursó solo los 3 primeros años en la escuela. El 38,9\% de las personas indígenas de 15 años y más de edad es analfabeto y apenas el $12,2 \%$ cuenta con seguro médico.

A partir de 1997, se desencadenó una profunda recesión económica. Al finalizar 2003 el PIB per cápita se situó $30,0 \%$ por debajo del correspondiente a 1991. Según la Encuesta Permanente de Hogares $2007^{3}$, 35,6\% de la población viven en condiciones de pobreza. Asunción es el área geográfica que posee una proporción más reducida de habitantes que vive en condiciones de pobreza $(27,6 \%)$, de los cuales más de la mitad en extrema pobreza.
Las principales causas de mortalidad en el Paraguay son: enfermedades del sistema circulatorio: $15,3 \%$; tumores: $14,1 \%$; enfermedades cerebrovascular: $9,1 \%$; enfermedades del aparato respiratorio: $6,0 \%$, causas externas, traumatismos y violencia: $5,7 \%$. Se suma a estos datos que el 1,2\% de la población muere por diarrea, $0,9 \%$ por tuberculosis, $1 \%$ de enfermedades nutricionales y anemia. Entre los niños de 1 a 4 años el $10,3 \%$ muere por causas externas como accidentes y traumatismos, $8,8 \%$ por neumonía e influenza, $7,7 \%$ por diarrea, $5,5 \%$ por enfermedades nutricionales y anemia ${ }^{4}$.

La morbilidad de los adultos está fuertemente asociada a la hipertensión arterial y la diabetes, existiendo también problemas de neoplasias, principalmente, mama y cuello uterino en la mujer, y los de próstata, tracto digestivo y pulmón en el varón.

Entre las causas de morbilidad en la población infantil se destacan las infecciosas, intestinales y respiratorias. La cobertura de vacunación en los últimos años no llegó a los valores considerados satisfactorios según la Organización Panamericana de la Salud, sin embargo, no hubo problemas relevantes con las enfermedades inmuno prevenibles 5 .

La protección en salud en Paraguay es segmentada. Los subsectores integrantes del sistema de salud incluyen como parte del sector público los servicios del Ministerio de Salud Pública y Bienestar Social (MSPyBS) y el seguro social con afiliación de los trabajadores formales al Instituto de Previsión Social, el subsector privado sin fines de lucro, privado con fines de lucro y mixto. Además de una alta segmentación de las coberturas entre estos subsectores, a nivel de los proveedores hay una desarticulación de los diferentes subsectores con fragmentación de la red asistencial. El MSPyBS desarrolla funciones de rectoría, provisión y financiamiento. El Instituto de Previsión Social desarrolla funciones de aseguramiento, provisión y financiamiento, pero los segmentos son autónomos y no existe articulación entre ellos. La cobertura de la Previsión Social abarca únicamente al 13,9\% y un 7,7\% están cubiertos por otros tipos de seguros privado o público, como individual, familiar, militar, poli$\mathrm{cial}^{3}$. El subsector privado se representa por instituciones con fines de lucro y sin fines de lucro. El primero incluye empresas de medicina prepaga, instituciones asistenciales con servicios de consultorios e internación, laboratorios y farmacias; con organización, funcionamiento y beneficios ofertados muy diversos. Las instituciones priva- 
Tabla 1. Indicadores demográficos, socioeconómicos y de salud, Paraguay, 2007*.

\begin{tabular}{|c|c|c|}
\hline & Indicadores & Paraguay \\
\hline \multirow[t]{4}{*}{ Demográficos } & Población & $6.054 .976(2007) \mathrm{d}$ \\
\hline & $<15$ años & $37,1 \%$ \\
\hline & $>60$ años & $7,1 \%$ \\
\hline & Esperanza de vida al nacer & $71,08(2007)$ \\
\hline \multirow[t]{7}{*}{ Socioeconómicos } & $\begin{array}{l}\text { Producto bruto interno (PBI) per cápita } \$ \text { internacionales } \\
\text { (ajuste PPA) }\end{array}$ & $1.362(2000-2005)^{\mathrm{e}}$ \\
\hline & Proporción de población bajo la línea nacional de pobreza & $35,60 \%(2007)^{d}$ \\
\hline & Proporción de población bajo la línea nacional de indigencia & $19,40 \%(2007)$ \\
\hline & Tasa media anual de inflación (\%) & $6,00(2007)^{\mathrm{a}}$ \\
\hline & $\begin{array}{l}\text { Proporción de la población con acceso sostenible a fuentes } \\
\text { mejoradas de abastecimiento de agua potable (población } \\
\text { urbana y rural) }\end{array}$ & $63,40(2002)^{b}$ \\
\hline & $\begin{array}{l}\text { Proporción de la población con acceso a servicios de } \\
\text { saneamiento mejorada (población urbana y rural) }\end{array}$ & $46,40(2002)^{b}$ \\
\hline & $\%$ de población alfabetizada $>15$ años & $93,90(2002)$ \\
\hline \multirow{11}{*}{$\begin{array}{l}\text { Mortalidad y } \\
\text { morbilidad }\end{array}$} & Tasa de mortalidad Infantil & $17,80(2006)$ \\
\hline & Tasa de mortalidad estimada en $<5$ años & $39,00(2006)^{8}$ \\
\hline & Razón de mortalidad materna & 108,00 \\
\hline & $\begin{array}{l}\text { Proporción anual de defunciones registradas de menores de } \\
5 \text { años por enfermedades infecciosas Intestinales (EDA) }\end{array}$ & $7,7 \%$ \\
\hline & $\begin{array}{l}\text { Mortalidad proporcional por enfermedades del sistema } \\
\text { circulatorio }\end{array}$ & $15,3 \%$ c \\
\hline & Mortalidad proporcional por neoplasias & $14,1 \%$ \\
\hline & $\begin{array}{l}\text { Mortalidad proporcional por enfermedades } \\
\text { cerebrovasculares }\end{array}$ & $9,1 \%$ \\
\hline & $\begin{array}{l}\text { Mortalidad proporcional por enfermedades del aparato } \\
\text { respiratorio }\end{array}$ & $6,0 \%$ \\
\hline & Mortalidad proporcional por causas externas & $5,7 \%$ \\
\hline & $\begin{array}{l}\text { Tasa de incidencia de tuberculosis baciloscopia positiva em } \\
100.000\end{array}$ & 37,40 \\
\hline & Tasa de incidencia de SIDA & 16,10 \\
\hline \multirow[t]{9}{*}{$\begin{array}{l}\text { Recursos, accesos } \\
\text { cobertura }\end{array}$} & $\begin{array}{l}\text { Proporción de población de } 1 \text { año de edad inmunizada } \\
\text { contra sarampión }\end{array}$ & $88,00(2006)^{f}$ \\
\hline & $\begin{array}{l}\text { Proporción depoblación gestante atendida por personal } \\
\text { capacitado durante el embarazo }\end{array}$ & $94,20(2004)$ \\
\hline & $\begin{array}{l}\text { Proporción de partos atendidos por personal capacitado- } \\
\text { institucionales }\end{array}$ & $84,8(2003)$ \\
\hline & RRHH por 10000 habitantes. Médicos & 6,0 \\
\hline & RRHH por 10000 habitantes. Enfermeras & 2,8 \\
\hline & Camas hospitalarias por 1000 habitantes & 0,9 \\
\hline & Gasto público en salud (proporción del PBI) & $2,7(2002-2004)^{e}$ \\
\hline & Gasto privado en salud (proporción del PBI) & $3,9(2002-2004)$ \\
\hline & $\%$ del gasto público en salud en el total de gastos de salud & $39,0 \%$ \\
\hline
\end{tabular}

* O año más reciente disponible.

Fuentes: a Banco Central del Paraguay; ${ }^{\mathrm{b}}$ MSPBS/OPS. Perfil del Sistema del Paraguay; ${ }^{\mathrm{c}}$ Dirección de Bioestadística. Dirección General de Planificación y Evaluación; ${ }^{\mathrm{d}}$ Encuesta Permanente de Hogares; 2007; ${ }^{\mathrm{e}}$ Cuentas de Salud 2002-2004. MSP y BS-OPS/ OMS. Informe Económico. BCP; ${ }^{\mathrm{f}}$ Organización Panamericana de la Salud. Salud en la Américas: Paraguay. Washington D.C. OPS/ OMS 2007; ${ }^{\mathrm{g}}$ Organización Panamericana de la Salud. Indicadores y datos básicos 2007. Washington D.C.: OPS/OMS; 2007.

das sin fines de lucro corresponden a organizaciones no gubernamentales y de otro tipo, algunas con financiamiento parcial del estado, como el caso del Hospital de la Cruz Roja Paraguaya. Esta organizaciones desarrollan muchas veces programas verticales y contribuyen para la frag- mentación, separando acciones de prevención y asistencia $^{6,7}$.

La segmentación de los sistemas de salud es una condicionante estructural de la fragmentación de los servicios, que se expresa de distintas formas en la organización y gestión. La segmen- 
tación se caracteriza por la existencia de subsistemas dirigidos a distintos segmentos de la población, cada uno operando de forma independiente, con su propia financiación, gestión y organización de los servicios ${ }^{8}$. De esto resulta la fragmentación, o sea, que cada subsistema crea y opera su propia rede de provisión de servicios de salud, con limitado grado de integración y comunicación entre los servicios que las forman - sea dentro de un mismo subsistema sea entre los subsistemas y los distintos niveles de atención?.

La baja condición de salud en Paraguay refleja la gran fragilidad del sistema. Además de la segmentación, hay una gran proporción de la población (cerca de $40 \%$ ) que no tiene acceso a servicios de salud. Existen evidencias de procesos de incorporación de algunos de los principios de la APS en las políticas sanitarias de los últimos 20 años, y la transición actual de un nuevo gobierno instaurado en el año 2008 explicita la incorporación de la estrategia en su política, la efectiviza e incorpora las denominadas "Unidades de Salud de la Familia" como puerta de entrada al sistema público de salud.

\section{Metodologia}

En este estudio se considera que la segmentación en el interior de los sistemas de salud y la resultante fragmentación de los servicios de salud en gran medida condicionan la concepción de APS implementada en cada contexto nacional específico, y, constituye ejes transversales para el análisis $^{1}$. Por otro lado se interroga si, ¿la implementación de la APS según el abordaje abarcadora es un elemento potencial para el reordenamiento del sistema hacia una mayor equidad y efectividad?; la APS abarcadora está expresa en los movimientos recientes de reforma del sistema de salud $^{10,11}$, coherente con las recomendaciones de la renovación de la APS en las Américas ${ }^{12,13}$. Esta concepción se contrapone a propuestas de focalización en la pobreza o de oferta de una canasta básica de servicios. Con bases en la concepción abarcadora de APS se estableció un modelo de análisis con 5 dimensiones:

(1) Rectoría de la APS - investiga las atribuciones, competencias e instrumentos de las esferas gubernamentales nacional y subnacionales en la formulación e implementación de la política en atención primaria en salud.

(2) Financiamiento de la APS - investiga el modelo de protección social presente en el país, las fuentes de financiamiento público (nacionaly municipal) y privadas para APS, los mecanismos y formas de transferencias intergubernamentales de recursos para APS.

(3) Recursos para la APS - investiga las instituciones responsables por la APS, tipos de servicios públicos y/o privados que brindan y a qué población se destinan, la oferta de servicios en APS, los profesionales de salud involucrados en los equipos de atención primaria, sus atribuciones, calificación, entrenamiento y cómo son contratados.

(4) Integración de la APS en el sistema de salud $y$ continuidad del cuidado - investiga la organización del sistema de salud en niveles de atención y el rol de los servicios de atención primaria, si los servicios de atención primaria los servicios de primer contacto, la articulación con otros servicios ambulatorios y hospitalarios del sistema de salud de mayor complejidad para asegurar la continuidad del cuidado.

(5) Articulación intersectorial y relaciones de la APS con actores sociales y políticos - investiga la interrelación con otros sectores que implementan políticas públicas para enfrentar los problemas de salud de la población y sus determinantes, la interrelación con la población para enfrentar a sus problemas de salud, la participación de la comunidad.

Con vistas a describir el sistema de salud y las características de la APS se analizó informaciones provenientes principalmente de la literatura y documentos oficiales (leyes y otros reglamentos). Complementariamente se entrevistó a 42 informantes claves: 12 expertos, 13 gestores, 8 profesionales representantes de los gremios y 9 miembros de organizaciones de la sociedad civil organizada, vinculadas a la salud directa o indirectamente. Estas categorías representan los principales stakeholders y sus testimonios contribuyen para producir mayor entendimiento de los hallazgos. Las entrevistas semi-estruturadas siguieron una guía, cubriendo todas las dimensiones del estudio, y se realizaran en cerca de una hora y media. Los actores seleccionados accedieron a formar parte de la investigación previa presentación, lectura y firma del Consentimiento Informado.

\section{Resultados y discusión}

\section{Rectoría de la APS}

Al largo de la historia, Paraguay ha tenido escasos y lentos procesos de reforma en el sistema de salud. Ellos ganaran importancia con las trans- 
formaciones políticas más recientes, con las cuales se inicia una serie de cambios estructurales y funcionales en el Sistema de Salud que reorientan el anterior modelo de atención, incorporando estrategias renovadas de Atención Primaria de Salud. De acuerdo con el documento "Políticas Públicas para la Calidad de Vida y Salud con Equidad" 4 , en cuyos enunciados se posiciona de manera central a la APS como eje programático y de gestión, se busca incrementar el acceso de la población excluida a servicios de salud de manera integral, en el marco de principios de equidad y universalidad, a través de equipos de salud de la familia, asignados a territorios sociales definidos.

El marco jurídico sanitario es representado, básicamente, por la Constitución Nacional de 1992, el código sanitario (Ley 836/80) ${ }^{14}$ y la Ley $1032 / 96^{15}$ que crea el Sistema Nacional de Salud en 1996. A ellos, se incorporan nuevas leyes y reglamentos que permiten implementar nuevos mecanismos administrativo financieros a fin de complementar los efectos derivados de la implementación de la gratuidad en todos los grupos de edad. Se destaca, entre otros, la "Ley 3007/ 2006 de Descentralización y sus reglamentaciones", que vincula el enfoque de derechos en salud con la participación comunitaria y la corresponsabilidad para la implementación de las políticas, planes, programas y proyectos vinculados a la salud y sus determinantes, y la creación de fondos de equidad y descentralización ${ }^{16}$ que hasta la fecha han llegado a 125 Consejos de Salud Descentralizados. Hay un reconocimiento de que se está pasando un proceso de reformulación con base en la APS: Las normas y reglamentaciones se están formulando a nivel del Ministerio de Salud, a través de la Dirección General de Atención Primaria de Salud (profesional 1).

La conducción de la APS en el nivel nacional, tiene el gran desafío de articular y coordinar el subsector público y privado, y es responsable de desencadenar procesos de capacitación continua y espacios de debate sobre lo que hay y lo que falta, las estrategias necesarias para cubrir la brecha de dotación de recursos humanos y la brecha de servicios de atención primaria.

...necesitamos generar la convicción de todos los actores [...] de instituciones, de profesionales de salud y sociedad [...] instalando el debate sanitario que hasta ahora ha sido pospuesto (experto 9).

...garantizar de manera explícita los derechos de los ciudadanos, que estos derechos se conozcan, se exijan, se cumplan (experto 9).

Hace falta reuniones que convoquen a universidades, ONGs, profesionales de la salud, para reins- talar el tema de APS, con apoyo de los documentos que se han generado en los últimos años. [Renovación de APS. Perfil del Sistema de Salud del Paraguay entre otros] con un lenguaje común y que hay cambios substantivos que se deben realizar en la docencia, en la investigación y en servicio con referencia al tema (experto 6).

En los niveles subnacionales, la conducción de la APS es ejercida por las autoridades sanitarias, apoyadas por las autoridades políticas a través de los consejos regionales y locales de salud, incluidos los intendentes, además de líderes comunitarios, profesionales y promotores o agentes comunitarios. Algunas contribuciones significativas de estas organizaciones mencionadas por gerentes entrevistados incluyen asesoramiento, formación de los promotores de salud, diseño de planes locales de salud, movilización de recursos y control ciudadano sobre la gestión pública.

Las dificultades reales para operacionalizar la APS, en los niveles subnacionales tienen que ver con restricciones presupuestarias, capacidad de gestión para formular políticas sanitarias descentralizadas, y organización incipiente.

Las autoridades locales son fundamentalmente facilitadores y copartícipes de la implementación de la estrategia de atención primaria en sus comunidades razón por la que es muy importante involucrarlos (sociedad civil 7).

El Consejo Local es un organización público privada a nivel local, que tiene a su cargo el diseño y la movilización de los programas de salud un órgano del cual participa el gobierno local, el intendente es presidente, junto a diferentes actores de la sociedad [...] busca atraer recursos de salud hacia el nivel local a través de la planificación, y el desarrollo de ciertos mecanismos de financiamiento (sociedad civil 6).

La figura de los Consejos Regionales y Locales de Salud, fue mencionada por diversos entrevistados como la instancia que aglutina a representantes de todos los subsectores y otros actores de relevancia vinculados a salud y sus determinantes sociales, económicos y políticos, integrando a actores claves y líderes comunitarios. Es el espacio donde la sociedad civil contribuye a la generación de planes, e instrumentos de conducción, en la mayoría de los casos incipientes, no formalizados oficialmente (sociedad civil 6).

Todavía permanecen amplios desafíos colocados para la conducción de la APS, desde de la articulación de los actores hasta la reformulación de la práctica. También en Brasil, en que existe un Sistema Único de Salud y la Estrategia de Salud de la Familia tiene más de una década de 
implementación, el proceso de cambio es gradual, y persisten problemas en la efectiva ejecución de la APS como un servicio de primer contacto y en la garantía de acceso a niveles más complejos de atención ${ }^{17}$.

\section{Financiamiento}

Los orígenes de las fuentes de financiamiento del sector salud son públicos, privados y externos. Provienen de las administraciones públicas (Administraciones Centrales, Departamentales y Municipales y el Instituto de Previsión Social), de los recursos del sector privado (Empresas privadas y los Hogares) y de los recursos externos de organismos de Cooperación Internacional. El Ministerio de Salud y el Instituto de Previsión Social son los dos grandes oferentes de servicios de salud.

El porcentaje del gasto público en salud, fue del 2,5\% del PBI per cápita y el gasto de bolsillo en salud representó aproximadamente el 3,9\% como proporción del PBI en promedio durante los años 2002-2004 ${ }^{18}$. El porcentaje de gasto público en salud está entre los más bajos de América Latina (39\%).

No existe una discriminación de lo que se gasta en APS en forma explícita en los análisis existentes. La oferta de servicios de salud es asumida mayormente por el Ministerio de Salud y por el Instituto de Previsión Social. El Ministerio de Salud además de dirigir la política sanitaria del gobierno presta servicios directamente. Sus recursos provienen del erario público, cobro de aranceles (copago) y de la cooperación internacional.

Los otros servicios públicos de salud corresponden a la Universidad Nacional de Asunción, financiada con cargo al Presupuesto General de Gastos de la Nación y con recursos propios; la Sanidad Militar y la Sanidad Policial financiadas con el Presupuesto General de Gastos de la Nación; las Gobernaciones también con ingresos del Presupuesto General de Gastos de la Nación con la obligación de destinar $10 \%$ a gastos sociales, aunque sin especificar lo que debe destinarse a salud.

En los últimos años se observó importante aumento del presupuesto del MSPyBS. El consolidado total del presupuesto del Ministerio de Salud Pública y Bienestar Social alcanzó, para el 2006, aproximadamente. 172 millones de dólares americanos y, en el año 2009, el equivalente a 375 millones de dólares.

No se han desarrollado sistemas de transferencias intergubernamentales en forma explícita hasta hace un año. Los mecanismos presupuestarios y las leyes administrativas no preveían mecanismos para ese efecto a pesar de los procesos de descentralización y desconcentración llevados adelante en los diferentes gobiernos que se sucedieron. En el marco de la Ley 1032/96 que creó el Sistema Nacional de Salud se planteó la creación del Fondo Nacional de Salud (FONASA), que se encargaría de la política de financiamiento del sector, sin embargo nunca fue reglamentado ${ }^{13}$.

\section{Recursos para APS}

Tradicionalmente, las actividades de APS se desarrollan en forma aislada en diferentes programas verticales, con escasa coordinación y articulación.

Los entrevistados reportan una brecha importante en la cobertura de APS.

Hay un porcentaje de personas que no tienen ninguna accesibilidad a la atención, consecuencia de las barreras, desde geográficas hasta todo el proceso cultural. Lo que ahora tenemos es una dispersión de servicios. [...] en Paraguay existe una cuestión que es el idioma, hay poblaciones que solamente hablan guaraní. Por otro lado están los indígenas, y su concepción del fenómeno salud enfermedad (gestor 7).

Los recursos y tecnología disponibles son insuficientes para responder a las demandas de la gente y se encuentran fragmentados por la segmentación del sistema, lo que profundiza la falta de acceso, la falta de oportunidad de atención, perdida de la continuidad en los procesos asistenciales y constituyen barreras a que los servicios respondan a las necesidades y expectativas de los usuarios.

Actores entrevistados relacionados al actual programa de atención primaria hacen referencia a la aplicación del Programa de Atención Primaria de Salud en el enfoque del nuevo gobierno, con la inclusión de las Unidades de Salud Familiar como puerta de entrada al sistema de salud.

Se han conformado 30 equipos de APS, en áreas de mayor necesidad como en el Bañado y la zona de San Pedro. Está previsto para el año que viene conformar 100 equipos más. [...] salarios más altos, 60 a $70 \%$ arriba de los salarios comunes. [...] se ha logrado lo máximo dentro de lo posible en el marco de la ley (gestor 1).

Los entrevistados apuntan el momento de implementación de la APS como estrategia transversal al sistema nacional, incluyendo atención de primer contacto por medio de equipos de proveedores integrados con un nivel de referencia 
que les permita afrontar condiciones que no pueden ser tratadas en el nivel primario. [Están involucrados] Los puestos sanitarios, la atención domiciliaria, las policlínicas y unidades sanitarias con atención medica y especialidades, el equipo móvil de atención de salud en las empresas etc. (experto 12). Están organizando los equipos de atención primaria que se centra en las familias en su territorio social real, con agentes comunitarios seleccionados por ellos mismos que tendrán la tarea de realizar en forma conjunta la recolección de datos e informaciones para la toma de decisiones y trabajar en forma coordinada con los diferentes actores sociales para fortalecer el acceso, el respeto al derecho de las personas, la aceptabilidad y la continuidad, que tienen relación directa con funciones o atributos considerados como específicos de la atención primaria.

La preocupación fundamental de los entrevistados referidos a los recursos humanos (RRHH) en APS, incluyen a proveedores, gestores, personal de apoyo y los trabajadores comunitarios que deben poseer los conocimientos, habilidades y destrezas adecuadas y tratar a las personas con dignidad y respeto pero que implica inversión a largo plazo, incluyendo capacitación, incentivos para mantener a las personas en sus puestos, así como las mejoras de las competencias en un proceso permanente. Hay un número insuficiente de trabajadores de salud calificados para proveer cobertura universal; su distribución en todo el sistema es desequilibrada o ineficiente. El trabajo en equipo está muy poco desarrollado o insuficientemente promovido. Los profesionales calificados prefieren trabajos en hospitales y ciudades, destacan los entrevistados. La incorporación de profesionales de manera a garantizar la continuidad de la relación médico/ paciente, es identificada como un obstáculo por investigadores de otros países, mismo que se adopte incentivos tales como la remuneración diferenciada ${ }^{19}$.

\section{Integralidad y continuidad}

La Política de Salud del actual gobierno nacional propone conformar una red que se inicia con la instalación progresiva de los primeros 100 primeros equipos de Atención Primaria en Salud; implementa progresivamente la red de urgencias y los sistemas de comunicación y transporte, así como la red de hospitalizaciones y la red de atención especializada entre otros ${ }^{4}$.

Las actividades asistenciales se realizan en los servicios, diferenciados por niveles de atención, conforme a un manual de organización que hasta el año 2008 preveía los niveles de Puestos de Salud y Dispensarios como las puertas de entrada y los niveles de Centros de Salud y Hospitales Distritales como centros de derivación primaria y luego a los hospitales distritales y finalmente a los hospitales de referencia nacional.

Desde fines del 2008 comienza la implementación de las Unidades de Atención de la Familia, dentro de la estrategia de cambiar la puerta de entrada con un médico de familia en este primer nivel de atención. También se prevé la derivación a otro nivel denominado "policlínicas" donde habrá consulta ambulatoria de especialidades. La atención complementaria con medicina alternativa no está prevista en los lineamientos de atención del sector público. En el sector privado existe un creciente mercado de este tipo de atenciones ${ }^{20}$.

Las actividades extramurales se circunscriben a acciones específicas para ciertos grupos de población sin acceso a los servicios de salud y dentro de algunos programas como por ejemplo el de inmunizaciones. La Atención Primaria de Salud se ha insertado desde su difusión, en los diferentes programas que el Ministerio de Salud ejecuta.

El análisis de la información recogidas de los entrevistados demuestra que algunos (especialmente gestores y expertos) apuntan los avances de la política actual y cómo se daría la integralidad y continuidad de los cuidados, mientras que otros manifiestan que aún es prematuro y que hay que darles fe siempre a todo proceso que se inicia, pero creo por lo menos los procesos que se iniciaron están dando algunos resultados (profesional 6). Pero hay unos entrevistados que poco refieren, tanto la Política como lo referente a los conceptos de integralidad y continuidad, sugiriendo que el nivel incipiente de implementación de la APS y la fragmentación del cuidado despiertan en ellos (especialmente representantes de la sociedad civil) otras preocupaciones que son anteriores a lo atendimiento de estos dos atributos de la APS.

El documento "Propuesta para el cambio 2008-2013"; documento guía de las políticas de salud de la actual administración, cita a la APS como puerta de entrada y lo integra a una red de servicios a crearse, incluyendo urgencias; proponiendo gratuidad al acceso universal de la salud, mecanismos de referencia y mejora en los mecanismos y participación de la población ${ }^{4}$.

Las referencias y contrareferencias no están estructuradas hasta la fecha a pesar de haberse realizado esfuerzos en ese sentido. Los lineamientos del manual específico estipulan reglas para 
referencia y contrareferencia que deberán ser observados en todos los servicios de salud del Ministerio de Salud para contribuir con ello a la reactivación del sistema en beneficio de la población ${ }^{21}$.

A través de las opiniones de los diferentes entrevistados, expertos, gestores, representantes de gremios de profesionales de la salud y de organizaciones de la sociedad civil se puede notar que en lugar de hablar o citar las acciones de APS hablan de la organización del sistema de salud que está comenzando a implementarse.

Una herramienta fundamental para la continuidad es la información. El Ministerio desde el año 1991 dispone de un sistema de información bioestadística que ha sido informatizado parcialmente y que contiene los módulos de estadísticas vitales y servicios prestados. Desde hace tres años se trabaja la modernización del mismo a través de la configuración de un sistema informatizado basado en la red de internet. $\mathrm{Al}$ efecto se cuenta con el apoyo de la cooperación internacional y se han desarrollado nuevos formularios que competen a las actividades de los servicios de atención médica en sus diferentes niveles, que a la fecha están en proceso de aprobación.

\section{Articulaciones intersectoriales y relaciones de la APS con actores sociales y políticos (stakeholders)}

La intersectorialidad, vista como la intervención coordinada de instituciones representativas de los sectores sociales, en acciones destinadas a tratar de atender y entender los problemas vinculados con la salud, es parte de la respuesta social organizada.

Consiste fundamentalmente en convertir la cooperación formal, no formal, eventual o casual en acciones, que lideradas por el sector de la salud y apoyadas en políticas nacionales o locales debidamente concertadas, se orienten estratégicamente hacia los problemas identificados y priorizados, donde las actividades de otros sectores pueden ser determinantes para el mejoramiento de los indicadores de salud.

La consigna es sumar la participación de los distintos sectores y trabajar en la construcción de alianzas estratégicas para fortalecer la defensa y el derecho a la salud.

En este contexto, los entrevistados han identificado que existen vínculos de relacionamiento de la APS con los responsables sectoriales departamentales o regionales y distritales o locales, mencionan el marco jurídico a partir del cual se ha impulsado la participación comunitaria y la coordinación intersectorial abordada en la Ley 1032/96 del Sistema Nacional de Salud, que posibilita la plena vigencia del derecho a la salud de toda la población ${ }^{13}$. La Ley del Sistema Nacional de Salud, además de definir la función de rectoría del Ministerio de Salud Pública y Bienestar Social, instituyó consejos de salud y define como componentes de la APS a la descentralización, la participación comunitaria e intersectorialidad.

El espacio formal de esa articulación es el Consejo Nacional de Salud, los Consejos Regionales de Salud y los Consejos Locales de Salud, donde confluyen instancias de gobierno de gobernaciones, de municipalidades, sociedad civil, y ciudadanos. Ese es el modelo formal de participación (experto 9).

Este acercamiento al ámbito local posibilita que la población, en su condición de sujeto y objeto, asuma la corresponsabilidad de su cuidado y forme parte del conocimiento de los problemas de salud y de las posibilidades de respuestas del sector.

Los actores sociales locales y la comunidad conocen la Ley pero existe un reclamo unánime de la falta de implementación y de confianza del ente Rector hacia las capacidades de gestión, administración, comprensión y resolución de los problemas de los Consejos de salud.

En el momento político actual el Gabinete social de la Presidencia de la República, constituye otra instancia de coordinación del Gobierno, a través de la cual se articulan acciones intersectoriales específicas, en el marco de programas y proyectos con los departamentos y distritos del país. Si bien el Gabinete Social ha sido instalado oficialmente a partir del año 2003, la percepción de los entrevistados apunta a reconocer que la madurez de los procesos ha llevado unos años, y que en este último Gobierno el mismo funciona de manera orgánica.

Yo creo que una de la innovaciones más importantes ha sido la comprensión de que los esfuerzos deben ser articulados [...] por primera vez funciona de manera orgánica un gabinete social, dependiendo del Presidente de la República, donde se definen justamente políticas articuladas para el sector social [...] de hecho el primer eje de las Políticas de Gobierno tienen que ver con lo social (experto 9).

Los gestores departamentales o regionales opinan que la intersectorialidad se está dando de una manera progresiva especialmente entre el ejecutivo y legislativo departamental y local, Intendencias y Consejos de las gobernaciones y municipios.

La sociedad civil organizada representada en los consejos locales de salud sean, organizaciones no gubernamentales, asociaciones de profe- 
sionales sin fines de lucro, comunidad constituida en Comités de Salud, o organizaciones de gestión privadas en salud convergen en que la intersectorialidad tienen avanzado.

La participación social está reglamentada por el decreto 22.385 promulgado el 14 de agosto del año 1998, sin embargo actores de la sociedad civil mencionan que los mecanismos de gestión participativa no son formales y responden a intereses mediáticos para satisfacer algunas urgencias de los servicios.

Claro, hay activa participación de la comunidad a través de la Municipalidad, pero las autoridades del Ministerio nos utilizan para pagar las cuentas que ellos no pueden cubrir (sociedad civil 3).

Se destacan acciones aisladas que con el grado de empoderamiento de las poblaciones se generaran compromisos y conductas ordenadas por la política.

De positivo se puede plantear que existe consenso y conciencia de la necesidad de acciones intersectoriales para poder enfrentar los problemas que atañen a la salud, pero se hace necesario pasar de las conceptualizaciones y los discursos, llenos de compromisos, metas, y buenas ideas, a las acciones concretas.

Las experiencias exitosas, o los modelos que han logrado avances con resultados manifiestos, podrían perfeccionarse y pasar de una relación entre los sectores coordinados y a veces inducidos, a una relación integrada, consciente e interactiva por la salud, el bienestar y la calidad de vida.

Para los actores entrevistados, los resultados en la intersectorialidad serán mayores en la medida que se logre descentralizar operativamente los procesos del sector y acercarlos, cada vez más, al ámbito local y comunitario; donde los sectores cuenten con el poder y libertad de acción suficiente para, conjuntamente, enfrentar los problemas, cuyas soluciones permitan alcanzar sus propósitos.

De igual forma, se reconoce en todas las entrevistas que las organizaciones a nivel local son el resultado de alianzas producto de la negociación continua que van cambiando las dinámicas del sector en una sociedad, localidad o grupo social.

El protagonismo que va logrando la población, en la participación comunitaria o social ${ }^{22}$, depende de la organización y conductas acertadas de los mismos, especialmente del sistema de trabajo como verdaderos equipos; solo así la población se apropiará de su condición de salud y podrá asumir con mayor responsabilidad, mayor interés, y confianza el cambio, termino presente en todos los discursos.
Así, la intersectorialidad, la participación social o comunitaria, la descentralización, las relaciones interinstitucionales palabras que fueron nombradas y entendidas en varias entrevistas por un mismo concepto se presenta como solución y problema al mismo tiempo, por consiguiente, entre las primeras tarea del ente rector del sector de la salud consiste en preparar a las fuerzas sociales para asumirla, pues sólo de esta forma, podrá lograr las transformaciones que se requieren en la forma de abordar los problemas que determinan o influyen en el bienestar de la población. El control social depende del interés y la movilización del usuario aliados a un sistema dispuesto a informar y mejorar su desempeño ${ }^{23}$.

\section{Consideraciones finales}

En Paraguay se está llevando a cabo una propuesta de reforma de la APS que acompaña reformas política más amplias y busca ser una estrategia que reduzca la inequidad y contempla la organización de los servicios, a través de la conformación de las unidades de atención primaria de salud.

Las principales debilidades identificadas en el estudio hacen relación, en primer lugar, a los Recursos Humanos, seguido de los Recursos Financieros, la rectoría por Ministerio de Salud Pública y Bienestar Social, en cuanto a la coordinación, normativas claras, organización, gestión y comunicación. Por otro lado, se destaca, el desconocimiento o concepto poco claro de la Estrategia APS; deficiencia en las instituciones formadoras e integración al Sistema Nacional de Salud.

Entre las principales fortalezas se destacan la actual condición política favorable, los recursos humanos engajados, las personas y las organizaciones que forman parte de las comunidades como un apoyo fuerte para el desarrollo de la APS, además el apoyo internacional que se está teniendo.

No tocante a perspectivas se destacan la necesidad de mayor aporte financiero y recursos humanos con adecuada capacitación, además de la adopción de un enfoque más programático. Pero, para que las mudanzas se efectúen son importantes la voluntad política, participación de la comunidad y de las organizaciones sociales comunitarias.

El cuadro actual permite una evaluación optimista del desarrollo futuro de la APS, que depende, también, del grado de priorización política y inversión financiera, del proceso de descentraliza- 
ción, de los avances en los mecanismos de capacitación de los recursos humanos, del apoyo y confianza de amplios sectores de la sociedad. Es necesario un esfuerzo que supere el escepticismo, generado por el histórico político del país. La implantación de la Estrategia Salud de la Familia, antes mismo de reformas estructurales en las instituciones puede ser una forma de rescate de confianza de los actores sociales, pues dedicase a enfrentar la precariedad de acceso a los servicios como el problema más urgente y prioritario.

La reorientación del sistema de salud hacia la APS requiere ajuste de los servicios hacia la promoción de la salud, asignación de funciones apropiadas para cada nivel de gobierno, integración de los servicios de salud públicos y personales, enfoque familiar y comunitario, y creación de un marco institucional con incentivos para mejorar la calidad de los servicios.

Para la adecuada formación de recursos humanos para la APS, las Universidades deben cambiar el perfil de salida, reformulando los contenidos, la metodología y técnicas didácticas que en este momento se centran en la formación de especialistas. Además, hay que desarrollar estrategias para mejorar y sostener la capacidad de los recursos humanos en el sector salud, mantener los profesionales motivados, supervisados y distribuidos de manera que sean capaces de realizar los elementos estructurales y funcionales de la APS.

Aun que no esté en la agenda política la constitución de un servicio nacional de salud con la unificación o articulación de los servicios del MSPyBS y del seguro social laboral (Instituto de Previsión Social), que incidiría sobre la segmentación, la expansión de la cobertura por equipos de salud familiar previstas en los planes guber- namentales actuales y en proceso de implementación, tiene potencial para responder a los retos actuales, logrando protección social en salud y prioridad para los grupos menos privilegiados, aumentando la salud y la equidad, perfeccionando la calidad, incrementando la participación y ampliando la responsabilidad del gobierno en la universalización del derecho a la salud.

\section{Colaboradores}

R Dullak y MI Rodríguez-Riveros participaron de la elaboración del diseño y la metodología, trabajo de campo, análisis y discusión de los resultados, y la redacción del artículo científico; I Bursztyn, de la elaboración del diseño y la metodología del estudio, análisis y discusión de los resultados, y de la redacción del artículo científico; MS Cabral-Bejarano, M Ruoti, ME Paredes, C Wildberger y F Molinas participaron del trabajo de campo, organización del banco de datos y análisis de los mismos.

\section{Agradecimientos}

A la Red de Investigación en Sistemas y Servicios de Salud del Cono Sur por el apoyo y al International Development Research Centre (IDRC) por la financiación de la investigación. 


\section{Referencias}

1. Bursztyn I, Kushnir R, Giovanella L, Stolkiner A, Sterman-Heimann L, Riveros M I., Sollazzo A. Notas para el estudio de la Atención Primaria en contextos de sistemas de salud segmentados. Rev Salud Pública (Bogotá) 2010; 12(Supl.1):77-88.

2. Paraguay. DGEEC. Principales resultados EHI/2008. Encuesta de hogares indígenas. Asunción: DGEEC; 2008.

3. Paraguay. DGEEC. Encuesta permanente de hogares. Asunción: DGEEC; 2007.

4. Alianza Patriótica para el Cambio. Propuesta de Salud: políticas públicas para la calidad de vida y salud con equidad en el Paraguay - 2008-2013. Asunción: La Alianza; 2008.

5. Banco Internacional de Reconstrucción y Fomento/Banco Mundial. Las prioridades de la salud proyecto sobre prioridades para el control de enfermedades. Washington, D.C.: Banco Mundial; 2006.

6. De Paepe P, Soors W, Unger JP. International aid policy: public disease control and private curative care? Cad Saude Publica 2007; 23(Supl.2):S273-S281.

7. Maciocco G, Stefanini A. From Alma-Ata to the Global Fund: the history of international health policy. Rev Bras Saude Mater Infant 2007; 7(4):479486.

8. Organización Panamericana de la Salud. Agenda de la Salud para las Americas, 2008-2017. Washington: OPAS; 2007.

9. Organización Panamericana de la Salud/Organización Mundial de la Salud [Internet]. Salud en las Américas. Washington DC, 2007. [documento na Internet]. [acessado $2011 \mathrm{fev} 25$ ]. Disponivel en: http://www.paho.org/hia/index.html

10. Giovanella L. A Atenção Primária à Saúde nos países da União Européia: configurações e reformas organizacionais na década de 1990. Cad Saude Pública 2006; 22(5):951-964.

11. Conill EM. Ensaio histórico-conceitual sobre a Atenção Primária à Saúde: desafios para a organização de serviços básicos e da Estratégia Saúde da Família em centros urbanos no Brasil. Cad Saude Publica 2008; 24(Supl.1):S7-S27.

12. Organización Panamericana de la Salud. Renovação da Atenção Primária em Saúde nas Américas: documento de posicionamento da Organização Pan-Americana da Saúde/Organização Mundial da Saúde (OPAS/OMS). Washington, D.C: OPAS; 2007.

13. World Health Organization. The World Health Report 2008: Primary Health Care (now more than ever). [documento na Internet]. [acessado $2011 \mathrm{fev} 25$ ]. Disponível em: http://www.who.int/whr/2008/en/ index.html
14. Paraguay. Ley $n^{\circ} 836 / 80$ del 15 de diciembre de 1980 del Código Sanitario del Congreso de la Nación Paraguaya. Registro Oficial 1980; 15 dic.

15. Paraguay. Ley n ${ }^{\circ} 1032 / 96$ del 18 de marzo de 1997, por la cual se crea el Sistema Nacional de Salud.; Registro Oficial1997; 10 oct.

16. Paraguay. Ministerio de la Salud Pública y Bienestar Social. Resolución SG 184 2008; 14 oct.

17. Escorel S, Giovanella L, Mendonça MHM, Senna, MCM. O Programa de Saúde da Família e a construção de um novo modelo para a atenção básica no Brasil. Rev Panam Salud Publica/Pan Am J Public Health 2007; 21(2):164-176.

18. Paraguay. Ministerio de Salud Pública y Bienestar Social, OPS, OMS. Cuentas de salud Paraguay 2002/ 2004. Asunción: OPS; 2006.

19. Mendonça MHM, Martins MIC, Giovanella L, Escorel S. Desafios para gestão do trabalho a partir de experiências exitosas de expansão da Estratégia de Saúde da Família. Cien Saude Colet 2010; 15(5):23552365.

20. Organización Panamericana de Salud. Perfil de los sistemas de salud de Paraguay: monitoreo y análisis de los procesos de cambio y reforma. Washington D.C.: OPS 2008.

21. Paraguay. Ministerio de Salud Pública y Bienestar Social. Manual de procedimientos para referencia y contrareferencia de pacientes. Asunción: Ministerio de Salud Pública y Bienestar Social; 2006.

22. Gohn MG. Empoderamento e participação da comunidade em políticas sociais. Saude Soc 2004 13(2):20-31.

23. Assis MMA, Villa TCS. O controle social e a democratização da informação: um processo em construção. Rev Latino-Am Enfermagem 2003; 11(3):376382.

Artigo apresentado em 18/10/2010

Aprovado em 20/11/2010

Versão final apresentada em 11/03/2010 